\title{
Implementation of a Student Lab Kit Case to Allow Interfacing with Online Laboratory Systems
}

\author{
Luis Felipe Zapata Rivera, MSc, Tri Nguyen, Bachelor, Vinh Huynh, Bachelor, Grant Kveton, Bachelor, and Lars \\ Koester, Bachelor \\ Florida Atlantic University, USA, lzapatariver2014@,fau.edu, tringuyen2014@,fau.edu, vhuynh2017@,fau.edu, \\ gkveton2017@,fau.edu, 1koester2015@,fau.edu
}

Mentor: Dr. María Mercedes Larrondo Petrie, petrie@fau.edu

\begin{abstract}
Online laboratories help students to perform laboratory assignments from different geographical locations, through the use of internet.

A possible configuration is the hybrid laboratories, that includes the use of a physical local experiment equipment, and a set of either virtual or remote laboratory components. One of the issues of this type of setups is how to determine whether the student is really performing the lab activities or she/he is presenting somebody else work. Therefore, it is important to take all the necessary steps to keep the students engaged while preventing academic dishonesty. This paper proposed a prototype of an Anti-Cheating student lab kit case, which can help to prevent plagiarism and cheating in the engineering laboratories. This case includes several measures to avoid dishonesty in engineering courses, such as software and hardware solutions. This paper will go into more detail of how the student lab kit case was designed and what challenges were faced during its implemenation.
\end{abstract}

Keywords-Laboratories, Anti-Cheating, 3-D Printing, Lab Kit Case, Software, Hardware, Mobile Laboratory, Hybrid Laboratory

\section{INTRODUCTION}

Physical laboratories can hinder the student's learning because of accessibility. Students may find it difficult to come to the lab to do a lab experiment. Due to its hindrance and inconvenience, online labs may be a solution for this situation. Online remote laboratories allow the students to access the lab remotely from anywhere through the access of internet. While it may be a solution to physical lab problems, it also brings some challenges. It can be problematic if the students decided to submit someone else work as their own. Therefore, this paper proposed a method to prevent academic dishonesty in online remote laboratories by designing an anti-cheating lab kit case.

The laboratory kit used in this paper was assembled and developed for Introduction to Logic Design in the department at Florida Atlantic University for Electrical Engineering, Computer Engineering and Computer Science by the Lab Manager Perry Weinthal. Introduction to Logic Design class will give the students their basic knowledge for their future courses, and the labs for this class will give them their first hands-on experiences with the knowledge learned in class instead of just the concepts. The type of student lab-kit that had been used for the class is a type of Mobile-Lab:

"Mobile laboratories refer to self-contained, traveling laboratories that accommodate student participation in hands-on laboratory science and engineering. They provide students and teachers with access to equipment and supplies that are difficult for most schools to acquire and maintain. However, mobile laboratory programs are designed to do much more than provide equipment and supplies. The value added by these programs stems from access to engaging, substantive, and thought-provoking inquiry experiences for students and teachers. These include a range of laboratory experiences that involve students carrying out carefully specified procedures to verify established scientific knowledge as well as experiences that engage students in formulating questions, designing investigations, and creating and revising explanatory models." [1]

With the idea of mobile lab in mind, The university also designed a common base unit for all labs and experiments for each lab. The idea of mobile lab allows the students to build their lab assignments on the go. It doesn't matter where they are, which means that they do not have to be in the laboratory room to complete and test their lab assignment. The provided lab-kit consists of: "a robust battery-operated power supply, reverse polarity protection for the logic buses, LED status indicators, more DIP-switches, more push button switches, Tri-State logic probe, dual 7-segment display, and dual square wave oscillators" [2]. Figure 1 shows a completed setup of the provided lab kit for the Logic Design course. 


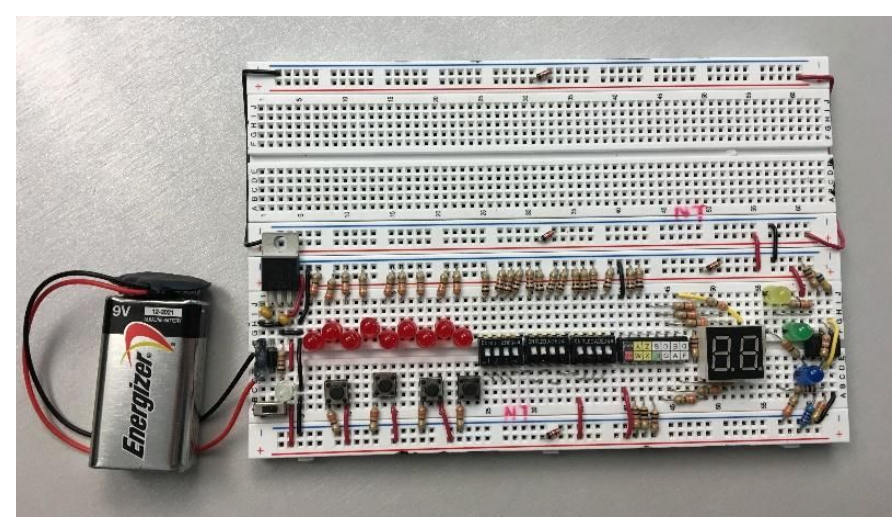

Figure 1: Breadboard Design by [2].

At first, the students could build their labs, and then they would come to the lab-room for help or grading from Teaching Assistant (TA). At that time, the only anti-cheating measurement was making the student write their initial name on their breadboard and the TA had to check for those names against the student name; on top of that, the student's breadboard had to be unwired after the lab experiment had been graded by the TA.

After that, another approach was implemented using remote laboratories, which will provide the student with more helping time from TA, by allowing the labs to be graded through computer via the remote lab system, by combining the provided Mobile Lab with a Saleae Analyzer [3]. In this approach, a Saleae Analyzer was used to get the digital signal from breadboard to computer, and the interface will generate the outcome base on provided signal [3]. However, there are a few drawbacks from this approach. The first drawback is that: "In order for this Program to work, the Saleae software must be running and the Saleae Analyzer must be connected to the computer" [3]. Then, the most important drawback is that there is a big loophole in anti-cheating measurement.

Now, this new implementation also applies the same idea from the previous approach, and it combines the idea of Mobile Lab with Remote Lab by combining the provided mobile lab with an Arduino Nano controller. This approach provides the developer more freedom in developing the software for the Nano Controller to give only the needed information from the breadboard; also the nano controller can be concealed easily, and the cost for Arduino Nano controller is cheaper comparing to the Saleae Analyzer. However, this approach also ran into a problem with anti-cheating measurement; one solution for this matter is assigning a special ID to each Nano controller and to check if it is associated with the system every time the students try to grade their labs. Besides that, it would be better to have a hardware anti-cheating solution for the breadboard itself since the labs will be graded online. The solution that will be discussed in this paper is creating a closed case that covers the Nano controller as well as the breadboard, which can't be removed after being put in and the student has built the basic setup for the provided lab kit; the case will break if the students forcefully try to remove the breadboard. The only way for the breadboard to be removed is by taking everything off the breadboard, which means after that the student will have to rebuild everything from the beginning.

\section{HyBRID LABORATORIES}

Laboratory experiments can be categorized into three main categories Physical, On-Line and Hybrid [4]. Physical Laboratory can be derived into smaller categories: On-Site and Mobile Lab; similarly, On-Line laboratory consists of Remote and Virtual Laboratories. The Hybrid laboratory can be a combination of two or more types of laboratories. This can be very useful, because it can be used in a wide variety of applications. Hybrid Laboratories are very important to education at all levels and especially so in post-secondary education. For example, a Hybrid lab could consist of on site and virtual labs. The relationship between the previously described categories of labs is illustrated in Figure 2.

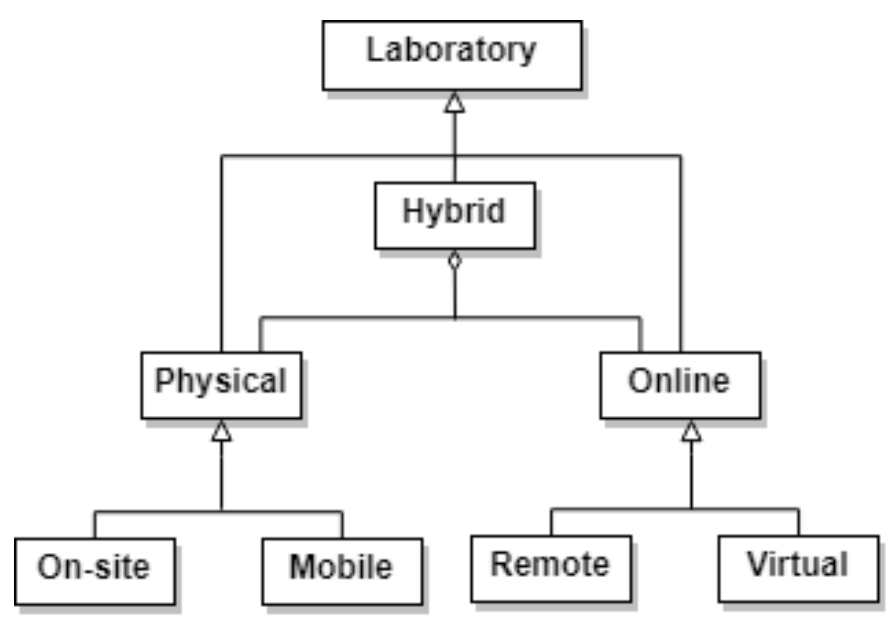

Figure 2. Proposed UML Model of Laboratory Taxonomy. [4]

\section{A. Remote Lab Architecture}

The architecture of the Smart Adaptive Remote Laboratory consists of different components interacting. These components include: the Virtual Learning Environment (VLE) [5] or the Learning Management System (LMS), the Learning Record Store (LRS) [6], the Remote Lab Management System (RLMS) [7], the Smart Adapter, the Remote Experiment, and the connection to the remote user [8]. These components are an important factor to the design and proper functionality of the Smart Adaptive Remote Laboratory. Their relationships are illustrated in Figure 3. 


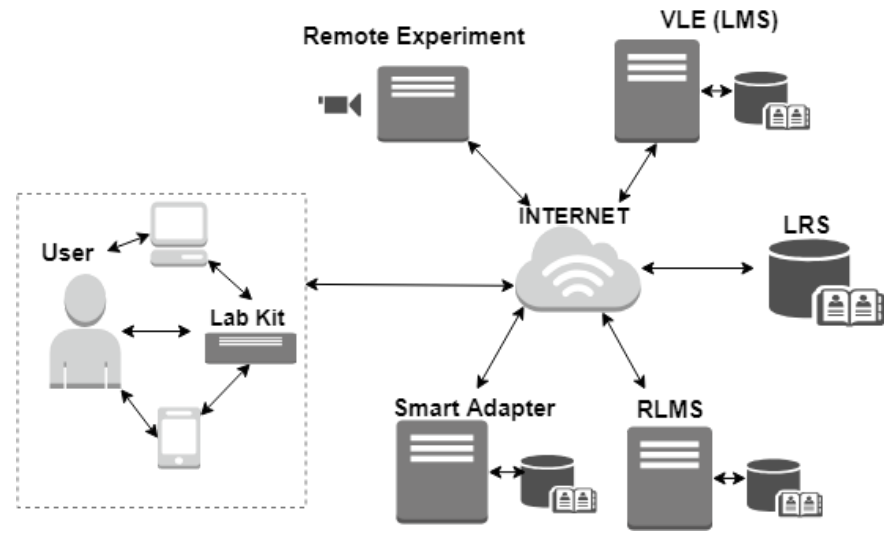

Figure 3. Architecture of the Remote Lab

The Virtual Learning Environment is a system allows academic information to be retrieved and allows for the user to interact with the laboratory experiment. The Learning Record Store is a database that holds data in a structured method. The Remote Lab Management System handles the remote experiments and the generation of user interfaces. The part of the Smart Adaptive Remote Laboratory system that most concerns this paper is the users connecting their experimental lab kit to a computer or device to access the remote lab experiments.

\section{B. Remote Lab Implementation}

The implementation shown in this paper is a Hybrid Laboratoty, this has eight configurations based on the different combination between Physical and On-Line lab: Local Access to Real Laboratory (on Site) with Online Access to a Virtual Laboratory, Local Access to Real Laboratory (on Site) with Local Access to a Virtual Laboratory, Local Access to a Mobile Laboratory and Online Access to a Virtual laboratory, Local Access to Mobile Laboratory with Local Access to a Virtual Laboratory, Remote Access to Mobile Laboratory and Online Access to a Virtual Laboratory, Remote Access to Mobile Laboratory and Local Access to a Virtual Laboratory, Remote Access to Real Laboratory (on Site) and Remote Access to a Virtual Laboratory, and Remote Access to Real Laboratory (on Site) and Local Access to a Virtual Laboratory [3].

The configurations used in this implementation are Local Access to Mobile Laboratory and Online Access to a Virtual laboratory. With this, the student will be able to build the experiments from their Mobile Lab kit at home or from anywhere that they feel comfortable and they can also test it on their own kit since the provided kit is a complete independent Mobile Lab Kit.
Once the student feels confident about their work, then they can connect the I/O ports from the nano controller to the breadboard. They have also to connect their controller to a laptop with an internet connection, and then login to the correct lab activity website that they wish to be graded on such as:

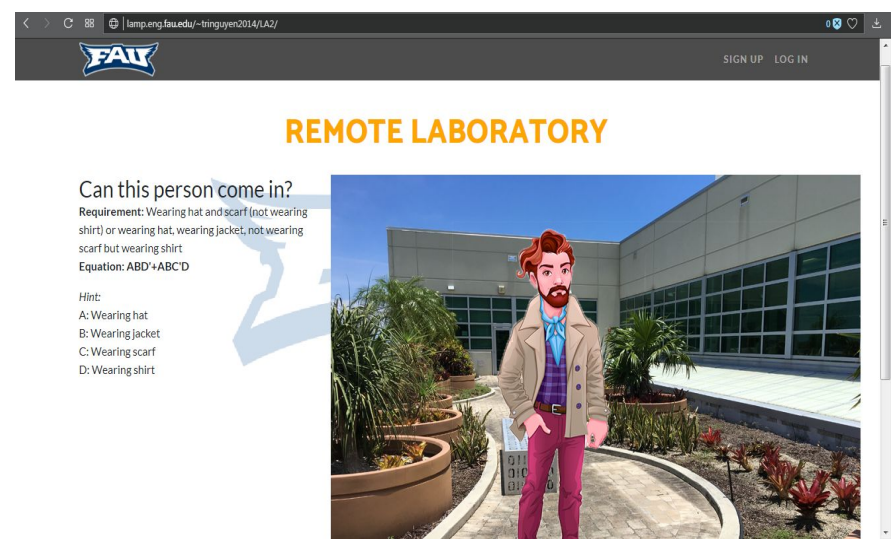

Figure 4. Example of Lab Activity Webpage.

Then the student can do the experiment on their breadboard according to the questions on the activity. The nano controller will send the digital signal generated from the breadboard to the interface. Finally, the interface will read the received signal and output the result to tell the student whether they did it correctly or not. At the end, the interface will also indicate the student's grade for the activity.

\section{RELATED WORKS}

One key component and aspect of remote lab development in education that must be considered is methods to prevent plagiarism and cheating between students. Within the study of remote laboratories anti-cheating can be described by three aspects:

\section{1) Personal presence of a given student (his or her authentication). \\ 2) Independent work during laboratory session. \\ 3) Authenticity of acquired data during the laboratory session." [9]}

The implementation of anti-cheating techniques in the proposition mostly describe methods for ensuring anti-plagiarism on the software side of development, but the concepts are also important to take into consideration for any hardware aspect of the remote lab that will require anti-cheating security. The paper also remarks the potential psychological implications of these anti-cheating precautions. Students are less likely to attempt to cheat or submit fraudulent data if security measures are in place from the start. 
The use of 3D printing is very important for developing prototypes within such a project. This allows for an ease of design for objects using software, and relatively cheap production costs. The impact of 3D printing in academic research is explained as:

"Many scientific research groups have a need for customized and specialized lab equipment which is sometimes hard to find or very expensive to buy. $3 D$ printers give them the luxury of easily acquiring their own equipment and build it directly by prototyping to save thousands of dollars in research funds." [10]

The process of prototyping and design of such a hardware case for this project was made much easier with access to the idea of prototyping with a 3D printer. The benefits of 3D printing in both academics, and a broader sense, are explained in the previously mentioned paper. With studies and applications in fields ranging from engineering to medical research.

One of the main challenges within the field of 3D printing is the cost of time consumption needed to create objects. [11] describes a proposed method of using a "novel additive process optimization algorithm for reducing the consumption time in 3D printing process". Such a topic may be out of the scope of the current stage of design and prototyping, but it is likely time consumption will need to be taken into account if multiple models need to be printed at the same time in the future.

The University of Kragujevac in Serbia conducted a case study and lab implementation involving a remote lab architecture. The lab was implemented with a hardware component and an online interface. Emphasis is put on the idea of problem-oriented engineering education, and that the implementation of remote labs will have a positive impact on problem-oriented education. It is concluded that the idea of remote labs is especially very important in education on a greater scale in terms of number of students, and that remote labs allow for easier access to lab equipment, with positive feedback from both students and teachers [12].

There are three main types of labs that can be found in engineering education: real or physical labs, virtual or on-line labs, and remote labs. Purely physical labs provide great hands on experience but face the limitations of time and scheduling required of both students and faculty, and virtual online labs provide easier accessibility to labs but are limited by a lack of hands on experience for students [13]. The disadvantages and benefits of each type of lab must be taken into account by the instructors when planning the course.

A case study of student's perceptions of remote labs was conducted at the University of South Australia. A survey that was taken in 2015 showed results that about half of the students had no preference to either the physical or remote labs. In an earlier survey taken in 2004, more than half of the students preferred the real physical lab over the remote lab. Although some students were shown to still prefer a physical lab, there was shown to be an overall positive perception regarding the convenience and accessibility of the remote lab [14].

\section{Development and Progress}

Other than preventing academic dishonesty in the laboratory, the case design also seek protection for the Nano processor and organize the student breadboard even more and to achieve a visually pleasing and efficient case structure. The research project started with just a sentiment of making an anti-cheating measurement for the remote lab. However, it ends up being another project in itself.

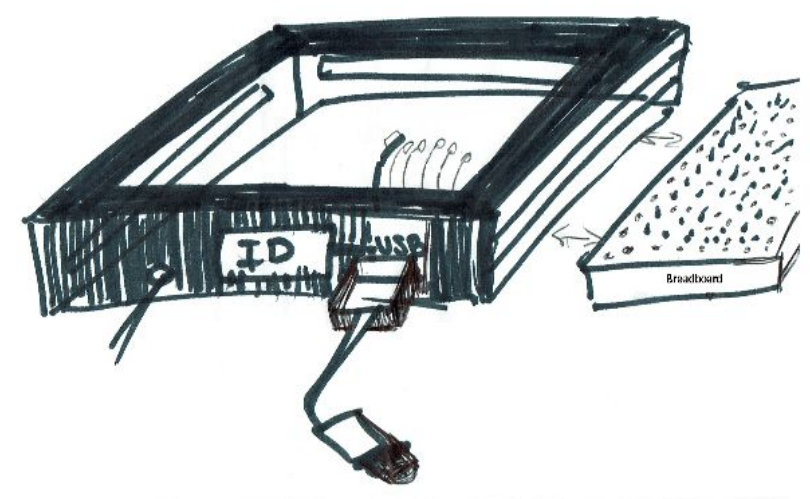

Figure 5: Original design (version 1)

In the original design, Fig. 5, the case was designed so that at the beginning of the course, the student can slide the breadboard in and out before they start their experiment. The height of the opening of the case and the height of the breadboard must be almost exact so once the students start building, the only way they can take the breadboard out is to remove all of the components from the breadboard. The position where the student slides their breadboard in was originally designed so they slide the breadboard in sideways. Unfortunately, that is not effective if the material for the case is not strong enough, the side of the case will likely to break than if the student is required to slide it in one end. 


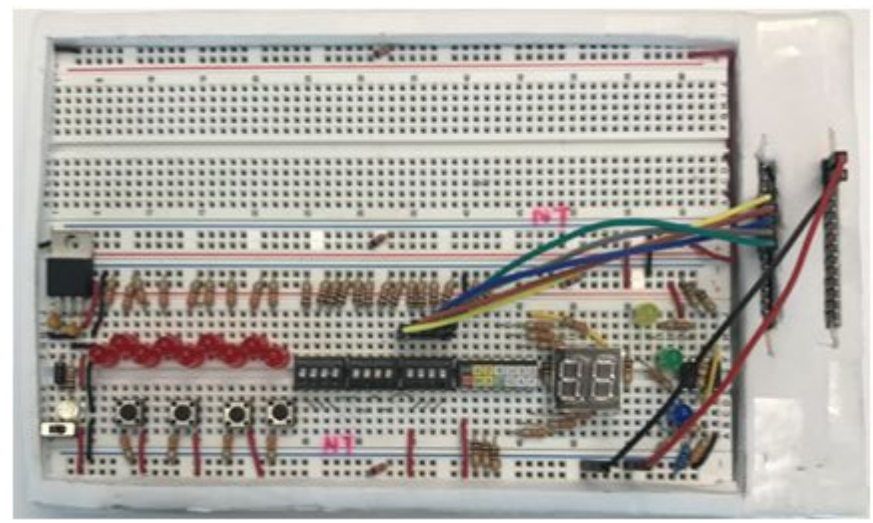

Figure 6: Version 1 Prototype

The version 1 prototype is shown in Fig. 6 . The student will slide the breadboard into the case from the left to right. The Nano processor will be placed on the right of the breadboard. It gets protection from the case itself on the right side and after the breadboard get pushed in, the left side of the Nano processor also get covered. Hence, the students cannot get access to the Nano processor once they have started the lab experiment.

Overall, version 1 is adequate, however, there is still room for improvement. Therefore, in the version 2 prototype, Fig. 7, the battery is also added to the case because there are still room next to the Nano processor. Both the Nano and battery are moved to the left of the breadboard since it is closer to where the student supposed to connect the battery into the breadboard. A battery holder is also designed so it will hold the battery down since the battery will fall out of its designated location if nothing is pushing it down.

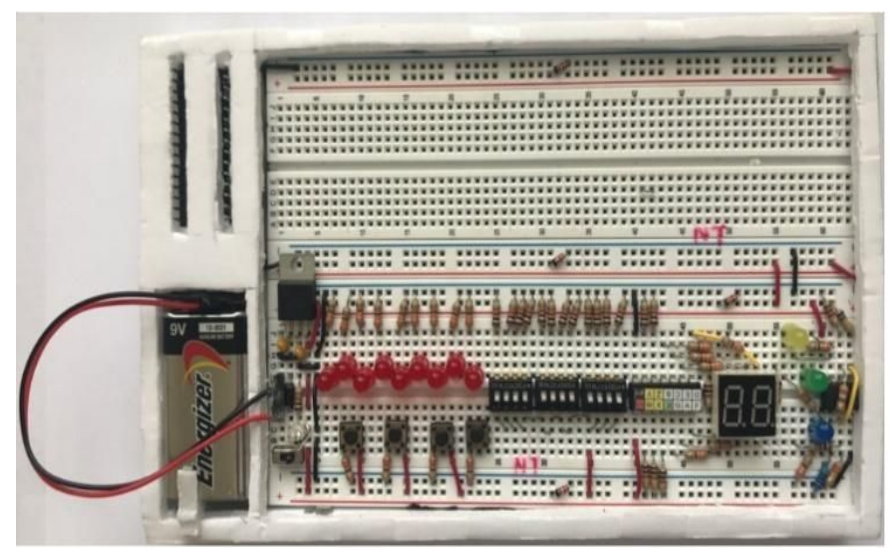

Figure 7: Version 2 Prototype

The battery holder did hold the battery in place, but there were a few problems with the holder. One being that students may have different sized batteries. Another issue was that, with the holder being a separate piece, it may be lost by the student.

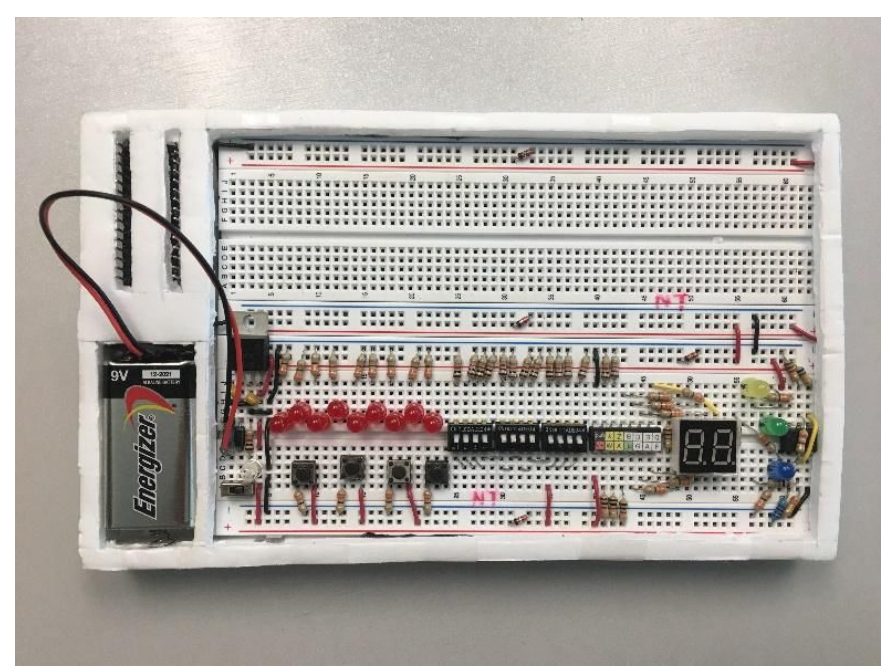

Figure 8: Version 2.1 with Spring

To solve the problem with the battery holder an idea similar to the way a battery is held in a remote controller with a spring was implemented. The spring allows for easier installation and removal of the battery. The spring implementation also allows for batteries of slightly different sizes to fit into the case. Figure 9 shows a closer look at the spring implementation of the prototype.

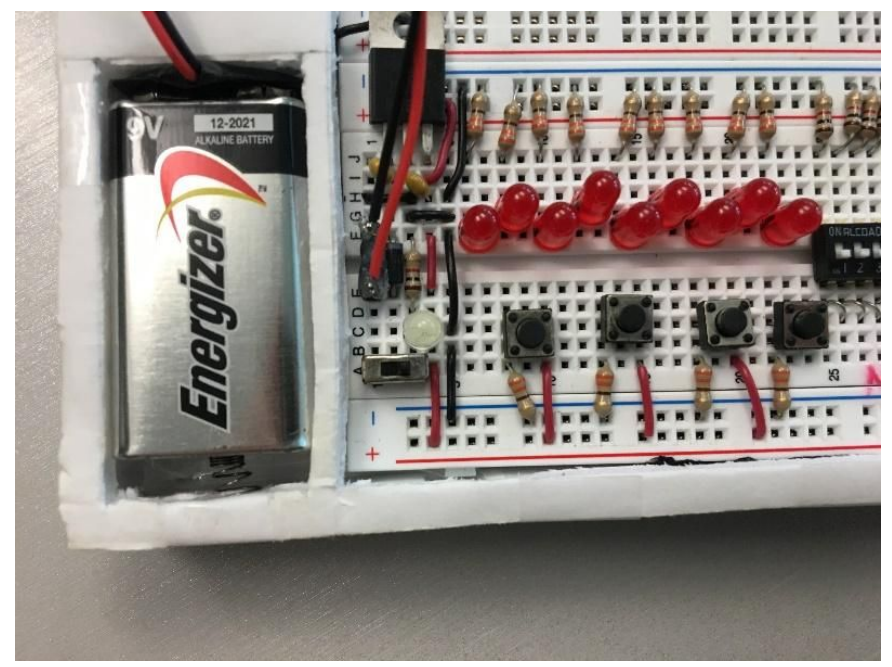

Figure 9: Improved Design with Spring

Figure 10 shows the 3D design model for Version 2.1 of the prototype. The piece on the bottom right of the picture is for when the student put their Nano processor in the top right of the case. That piece is to seal of the Nano before the students put their breadboard in. The 3D design for the breadboard is where the research is currently at in the design and production process.

It was discovered that due to the methods of 3D printing, the case may have to be printed into two separate parts that 
will later be connected. 3D printing is very similar to a hot glue gun, and because of this, the part that does not have support will collapse during the $3 \mathrm{D}$ printing process. This could be done by implementing pegs and slots into the $3 \mathrm{D}$ design before printing. In the following figure, all parts of the breadboard case prototype 2.1 are shown, except for the spring. The spring will be created separately from the $3 \mathrm{D}$ printed case and will be hot glued into the case.

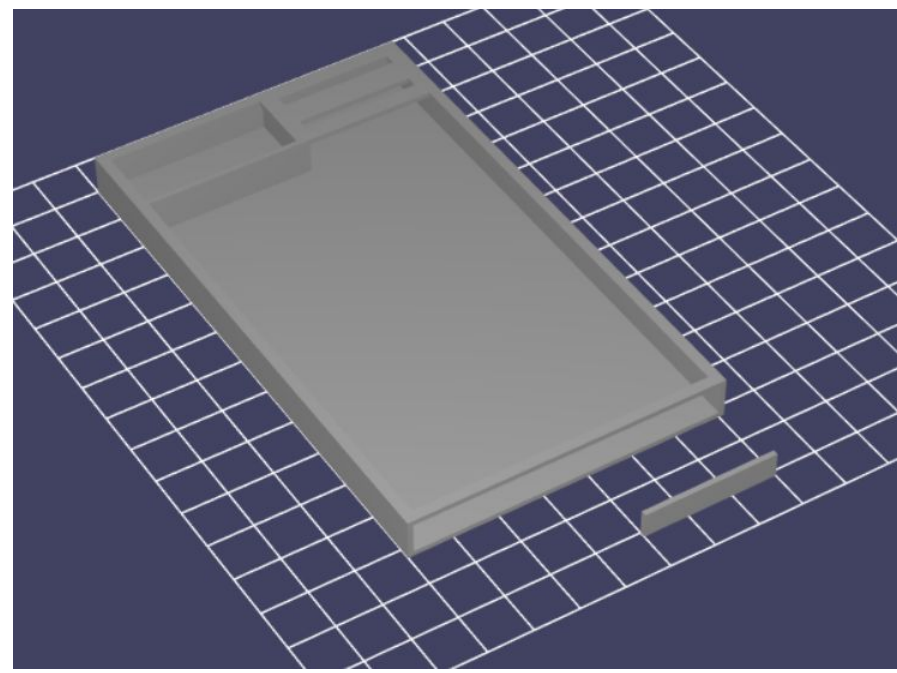

Figure 10: 3D design for Version 2.1

\section{FUTURE WORK}

In order to perfect the design of the lab kit case, there are testing, and small adjustment needed to be done. For instance, the battery holder is not professional when you first look at it; therefore, the research team wanted to design a lit that will keep the battery in place while making the case look professional at the same time. Testing for the material that is being used for the $3 \mathrm{D}$ printing is also important since it will decide what the thickness of the case will be. It would be beneficial to design the 3D printed case with square cut out of the bottom of the case, which would save on amount of material used. There also is an idea to modify the base design of the student breadboard kit to separate input and output.

\section{REFERENCES}

[1] C. Franzblau, C. A. Romney, R. Faux and D. DeRosa, "Mobile laboratory programs as vehicles to promote STEM education in K-12 and beyond," 2011 Frontiers in Education Conference (FIE), Rapid City, SD, 2011, pp. T3D-1-T3D-6.

[2] P. Weinthal, "Enhanced Tool Kit for Modular Portable Lab Kit for Logic Design", 2018 16th LACCEI International Multi-Conference for Engineering, Education, and Technology: "Innovation in Education and Inclusion”, 19-21 July 2018, Lima, Peru.

[3] T. Nguyen, L. Koester and A. Saint-Juste, "Logic Design for Physical and Virtual Laboratories," 2017 15th LACCEI International Multi-Conference for Engineering, Education, and Technology: "Global
Partnerships for Development and Engineering Education", 19-21 July 2017, Boca Raton Fl, United States.

[4] L. F. Z. Rivera and M. M. Larrondo-Petrie, "Models of remote laboratories and collaborative roles for learning environments," 2016 13th International Conference on Remote Engineering and Virtual Instrumentation (REV), Madrid, 2016, pp. 423-429.

[5] Y. Matsubara; S. Toihara; Y. Tsukinari. "Virtual learning environment for discovery learning and its application on operator training". IEICE Transactions on Information and Systems, 02/1997, Volume E80D, Issue 2

[6] H. D. Wuttke, M. Hamann and K. Henke, "Learning analytics in online remote labs," 2015 3rd Experiment International Conference (exp.at'15), Ponta Delgada, 2015, pp. 255-260.

[7] Remote Laboratory Management Systems and the iLab Shared Architecture (ISA) [Online]. Available: http://tempusdesire.eu/meetings/2015-04_summerschoolilmenau/downloads/presentations/Introduction\%20to\%20RL MS\%20and\%20ISA.pdf

[8] L. F. Z. Rivera, M. M. Larrondo-Petrie and L. Ribeiro Da Silva, "Implementation of cloud-based smart adaptive remote laboratories for education," 2017 IEEE Frontiers in Education Conference (FIE), Indianapolis, IN, 2017, pp. 1-5.

[9] I. Titov, A. Glotov, D. Bondarenko and S. Savkin, "Antiplagiarism in educational remote laboratories done right: Technological, educational and psychological implications," 2014 IEEE Global Engineering Education Conference (EDUCON), Istanbul, 2014, pp. 515-518.

[10] N. Bharti, S. Gonzalez and A. Buhler, "3D technology in libraries: Applications for teaching and research," 2015 4th International Symposium on Emerging Trends and Technologies in Libraries and Information Services, Noida, 2015, pp. 161-166.H. Simpson, Dumb Robots, 3rd ed., Springfield: UOS Press, 2004, pp.6-9.

[11] R. C. Luo, Y. W. Perng and P. K. Tseng, "3D printing process for multi-heterogeneous objects fabrication," 2017 IEEE/SICE International Symposium on System Integration (SII), Taipei, 2017, pp. 95-101.B. Simpson, et al, "Title of paper goes here if known," unpublished.

[12] M. S. Matijević, N. D. Jović, M. S. Nedeljković and Đ. S. Čantrak, "Remote labs and problem oriented engineering education," 2017 IEEE Global Engineering Education Conference (EDUCON), Athens, 2017, pp. 1391-1396.

[13] Z. Nedic, J. Machotka, and A. Nafalski, "Remote laboratories versus virtual and real laboratories," in Frontiers in Education, 2003. FIE 2003 33rd Annual, vol. 1. IEEE, 2003, pp. T3E-1.

[14] M. Teng, Z. Nedic and A. Nafalski, "Students' perception of remote laboratories - Case study: NetLab," 2016 IEEE Global Engineering Education Conference (EDUCON), Abu Dhabi, 2016, pp. 568-575.

16 $^{\text {th }}$ LACCEI International Multi-Conference for Engineering, Education, and Technology: "Innovation in Education and Inclusion", 19-21 July 2018, Lima, Peru. 